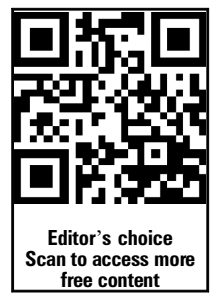

- Additional material is published online only. To view please visit the journal online (http://dx.doi.org/10.1136/ archdischild-2013-305349).

Department of Rheumatology, The Children's Hospital of Philadelphia, Philadelphia, Pennsylvania, USA

\section{Correspondence to}

Dr Elaine S Ramsay, Department of Rheumatology, The Children's Hospital of Philadelphia, 34th and Civic Center Blvd, Philadelphia, PA 19104, USA; RamsayE@email.chop.edu

Accepted 21 August 2014 Published Online First

9 September 2014

\section{CrossMark}

To cite: Ramsay ES, Lerman MA. Arch Dis Child Educ Pract Ed 2015;100: 30-36.

\title{
How to use the erythrocyte sedimentation rate in paediatrics
}

\author{
Elaine S Ramsay, Melissa A Lerman
}

\begin{abstract}
The erythrocyte sedimentation rate (ESR) has become a ubiquitously used technique in medicine as a marker of systemic illness. The test involves placing anticoagulated whole blood into an upright test tube and monitoring the rate at which red blood cells (RBC) fall over time. Negative charges keep RBC from sticking together. If this charge is neutralised, RBC stack into chains, or rouleaux, and fall more rapidly. ESR can be measured with a variety of tests: Westergren and modified Westergren; Wintrobe; micro-ESR. The Westergren is the most commonly used method of performing the ESR. Technical factors, such as temperature, time from specimen collection, tube orientation and vibration, can affect the results. RBC size, shape and concentration impact the ESR. Plasma characteristics are also important determinants of the ESR. Other factors that can change ESR include age, sex, race, medications and disease states, such as obesity, hypofibrinogenaemia and congestive heart failure. Other acute-phase reactants besides the ESR include C-reactive protein, fibrinogen, complement, ferritin, plasma viscosity, serum amyloid $A$ and albumin. When clinical suspicion for infection or inflammation is low, a normal ESR can reassure that there is no active disease. The slow rise ( $48 \mathrm{~h}$ ) and fall of the ESR relative to other acute-phase reactants may make it superior for monitoring inflammation in more chronic conditions. In conjunction with physical findings and other laboratory values, the ESR value can be used to screen for disease or disease complications, aid in disease diagnosis or assess disease activity or response to therapy.
\end{abstract}

\section{INTRODUCTION}

The erythrocyte sedimentation rate (ESR) has become a ubiquitously used technique in medicine. Clinicians in all specialties use the test, which measures the rate at which red blood cells (RBC) fall in a test tube, as a marker of systemic illness. The concept of using RBC sedimentation as a correlate for illness dates back to the Greeks. In the 19th century, a
Polish physician named Edmund Faustyn Biernacki published the first description of what has become the modern laboratory technique for the ESR. ${ }^{1} \mathrm{He}$ explained that blood with small numbers of RBC (anaemia) sediments faster than blood with higher numbers of $\mathrm{RBC}^{2}$; the level of plasma fibrinogen affects RBC sedimentation; and, because fibrinogen levels are increased in febrile diseases (eg, rheumatic fever), sedimentation rate increases. Publications by Robert Sanno Fahraeus followed, examining the utility of measuring ESR as a pregnancy test. ${ }^{2}$ He described factors that affect RBC agglutination and the correlation of RBC sedimentation with disease activity. Nearby, another Swedish internist, Alf Vilhelm Albertsson Westergren, studied RBC sedimentation in patients with tuberculosis. $^{2}{ }^{3} \mathrm{He}$ developed the Westergren method in 1920 and was responsible for standardising the ESR test that is still widely used today. Interestingly, in Europe the ESR test is commonly referred to as the FW test (Fahraeus-Westergren).

Systemic inflammation occurs in infection, autoimmunity, autoinflammatory conditions and malignancy. It can be tested for by numerous methods, including direct and indirect (surrogate) markers of inflammation. More direct measures include $\mathrm{C}$-reactive protein (CRP), fibrinogen, complement proteins, ferritin, plasma viscosity, serum amyloid $A$ and albumin (see box 1). The most commonly used is CRP, which is made in the liver in response to interleukin (IL)-1 $\beta$, IL-6 and tumour necrosis factor- $\alpha$. It activates neutrophils, inhibits platelets, aids in natural killer cell cytotoxicity and directs cells to the sites of inflammation by increasing adhesion molecule expression. ${ }^{1}$ Rather than directly measuring an acute-phase reactant, the ESR serves as a surrogate measure of 
Box 1 Other acute-phase reactants ${ }^{14 !}$

Positive acute-phase reactant (rise with disease activity)

- C-reactive protein

- Complement*

Ferritin

Fibrinogent

- Serum amyloid $A$

- Plasma viscosity

Negative acute-phase reactant (fall with disease activity) - Albumin

*Except in conditions with immune complex formation and subsequent complement depletion.

tExcept in macrophage activation syndrome.

inflammation, and it is affected, but not controlled, by plasma constituents such as fibrinogen (see table 1). For further comparison between ESR and CRP, see box 2 . The ESR is frequently used for screening, diagnosis, disease activity monitoring and as a prognostic tool for disease.

While there are various methods by which the ESR is performed, all involve placing anticoagulated whole blood into an upright test tube and monitoring the rate at which $\mathrm{RBC}$ fall over time. Because they are denser than plasma, RBC in whole blood settle to the test tube's bottom. The ESR is calculated by measuring the distance from the top of the tube to the meniscus of the settled RBC after a fixed period of time, in millimeters per hour (see figure $1 \mathrm{~A}-\mathrm{C}$ ).

\section{PHYSIOLOGICAL BACKGROUND}

The ESR is a marker of inflammation measured in vitro in a test tube, rather than a test of an in vivo phenomenon. As RBC fall in the tube, the downwards force of gravity is countered by an upwards force exerted by the viscous plasma. These forces are relatively equal when $\mathrm{RBC}$ remain as individual cells, but when agglutinated, RBC descend downwards. Anything that increases the agglutination of the RBC increases the ESR. RBC normally repel each other due to the negative charge conferred by cell surface syalic acid (zeta potential). If this charge is neutralised, RBC stack into chains, or rouleaux, and fall more rapidly.

The ESR has three phases: rouleaux formation, rapid fall and slow fall. During rouleaux formation, RBC are pulled together weakly by van der Waals

Table 1 Plasma characteristics affecting the erythrocyte sedimentation rate ${ }^{2} 45 \mathrm{w} 1$

\begin{tabular}{ll}
\hline Decreased & Increased \\
\hline Elevated albumin & Decreased albumin \\
Decreased fibrinogen & Elevated fibrinogen \\
Hyperviscosity & Globulins \\
\hline
\end{tabular}

Box 2 Why do I need to order an ESR and CRP at the same time?

CRP

- Directly measures the levels of a plasma protein in the blood (unlike ESR)

- Rises within 4-6 $\mathrm{h}$ of the onset of inflammation ${ }^{1}$

- Falls more quickly than ESR, often within $24 \mathrm{~h}$ of the initiation of treatment, ${ }_{1}^{1}$ if not, by $48 \mathrm{~h}$

- Can be run STATWide reference ranges (must be drawn several times to get useful values)

- Useful in diagnosing acute infection because tighter correlation with acute inflammation ${ }^{8}$

- Screens for septic joints: sensitivity is higher $(90 \%$ vs $66 \%$, respectively) while specificity is lower (15\% vs $48 \%)^{4} 9$

- Monitors disease activity for acute processes: resolution of meningitis, healing after surgery or major burns.

ESR

- Surrogate measure of inflammation

- Rises in 24-48 $\mathrm{h}$ and lags behind the resolution of inflammation.

- Slower decline may more closely parallel the complete resolution of inflammation.

- Test takes a minimum of an hour to result

- Rises in SLE flares, and the CRP is often unaffectedsuspect infection, rather than SLE flare, when the CRP is elevated. ${ }^{10}$ Measure them at the same time when faced with a sick patient with SLE.

CRP, C-reactive protein; ESR, erythrocyte sedimentation rate; $S L E$, systemic lupus erythematosus.

forces (first $10 \mathrm{~min}$ ). Over the next $40 \mathrm{~min}$, the interaction with other plasma macromolecules stimulates greater agglutination, and RBC fall rapidly. During the last stage, the rate decreases as RBC accumulate at the bottom of the tube. ${ }^{11}$ The ESR rises within $24-48 \mathrm{~h}$ of the onset of inflammation and when it falls, it lags behind the resolution of inflammation.

RBC size, concentration and shape can affect the ESR. Larger RBC have a lower surface/volume ratio and settle faster. Anaemia with a haematocrit lower than 20\% increases the ESR. This may be because there is less slowing of rouleaux descent by cells piling at the bottom of the tube ${ }^{12}$ and/or rouleaux formation increases when RBC are less concentrated. ${ }^{8}$ Conversely, in polycythaemia, the rouleaux are less compact and the ESR may be falsely decreased. Molecular asymmetry (eg, sickle cells, spherocytes) interferes with aggregation of RBC and lowers the ESR. ${ }^{8}$ Haemolytic anaemia leads to abnormally low ESR, potentially because the presence of large amounts of antibody can interfere with agglutination (see table 2). ${ }^{8}$ While formulas have been created to 


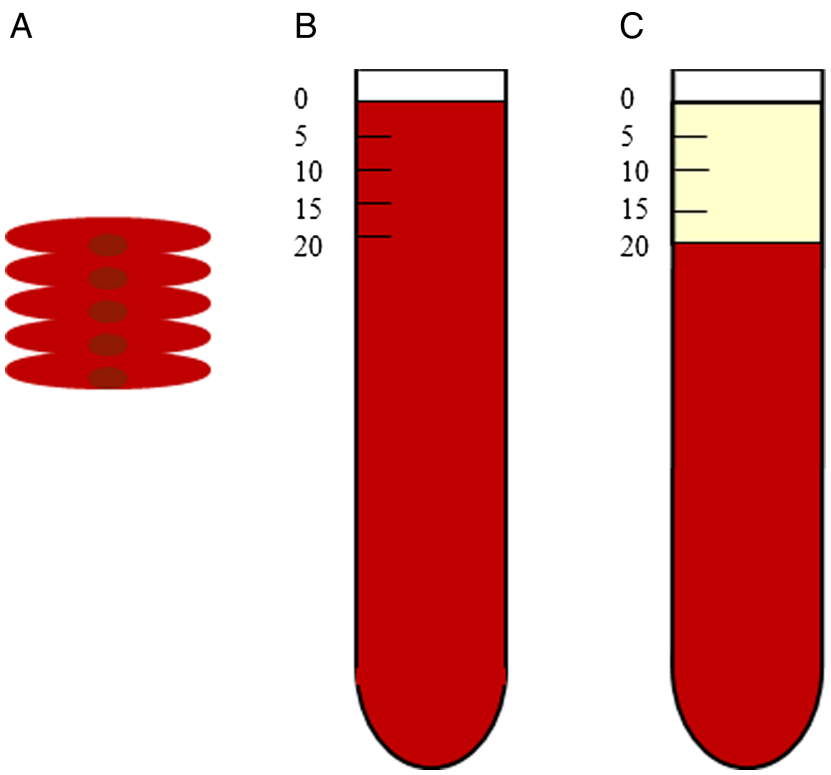

Figure 1 (A) Rouleaux of red blood cells. (B) Test tube with anticoagulated whole blood at time 0 h. (C) Erythrocyte sedimentation rate of $20 \mathrm{~mm} / \mathrm{h} 1 \mathrm{~h}$ later, plasma at top of tube.

correct the ESR for anaemia, none have been generally accepted.

In addition to being affected by RBC characteristics, the rate of settling is affected by plasma proteins. Serum proteins, such as fibrinogen and globulins, are positively charged and serve to overcome the negative repulsion between $\mathrm{RBC}$ by neutralising the negative zeta potential charges on RBC, and hence greatly contribute to rouleaux formation resulting in high ESR. Of these, fibrinogen is the largest contributor to agglutination, followed by $\gamma$ globulins, which have a lower capacity to induce agglutination. ${ }^{4}$ As their names imply, hypofibrinogenaemia of the newborn or congenital hypofibrinogenaemia lead to decreased ESR. ${ }^{4}$ Albumin is thought to break up rouleaux and slow down red cell aggregation, resulting in a lower ESR (see table 2). ${ }^{9}$ While this mechanism has been proposed to explain the correlation between clinical hypoalbuminaemia and elevated ESR, in vitro studies have demonstrated that the presence of other factors (fibrinogen, immunoglobulins) impacts the relationship between albumin and ESR. ${ }^{13}$

The utility of the ESR as a measure of inflammation is often attributed to its correlation with serum levels of fibrinogen, an acute-phase reactant. However, the

Table 2 Red blood cell characteristics affecting the erythrocyte sedimentation rate $245712 \mathrm{w} 1$

\begin{tabular}{lll}
\hline & Decreased & Increased \\
\hline Size & Microcytosis & Macrocytosis \\
Number & Polycythaemia & Anaemia \\
Shape & Sickle cell disease hereditary spherocytosis & \\
Destruction & Haemolytic anaemia & \\
\hline
\end{tabular}

relationship between ESR and inflammation is more complex as numerous studies have failed to show a direct correlation between ESR and fibrinogen. If fibrinogen serves as a major factor affecting ESR, the ESR may partially reflect inflammatory cytokine levels, but it does not directly measure these.

\section{TECHNOLOGICAL BACKGROUND}

\section{Methods}

There are numerous methods by which the ESR can be performed, including the Westergren and modified Westergren, Wintrobe and micro-ESR. The Westergren is the most commonly used method of performing the ESR. ${ }^{8}$ It is performed by mixing $2 \mathrm{~mL}$ of whole blood with $0.5 \mathrm{~mL}$ (or a quarter volume) of a sodium citrate solution, which is then used to fill a $200 \mathrm{~mm}$ pipet. The pipet is placed in a vertical rack for $60 \mathrm{~min}$. The long tube minimises clumping of RBCs to prevent false increases in the ESR. When EDTA is used instead of sodium citrate, the test is referred to as the modified Westergren (commonly used by commercial laboratories). This allows for blood to be stored up to $4 \mathrm{~h}$ at $4^{\circ} \mathrm{C}$ and for use of saline diluted blood employed for other studies, without changing the ESR. The International Committee for Standardization of Hematology prefers the Westergren method using whole blood that has been anticoagulated with EDTA, then diluted with sodium citrate or saline, or whole blood anticoagulated with sodium citrate as a reference for determining the ESR. ${ }^{14}$

Another frequently used method, the Wintrobe method, uses a $100 \mathrm{~mm}$ column with dry oxalate anticoagulant, no dilution and corrects for anaemia. ${ }^{8}$ Because the blood is not diluted, the same tube can be 
used to determine the haematocrit. The tube is shorter than that used in the Westergren method, which can lead to a high plasma viscosity and thus decrease ESR. The lack of dilution by citrate limits the potential of falsely decreasing the ESR as in the Westergren method. Low haematocrit will falsely increase the ESR using this method as well, whereas the Wintrobe method incorporated a technique to correct for anaemia, although a controversial one. Overall, while the Wintrobe method is more sensitive to small increases in the ESR above the normal value than the Westergren method, it is insensitive to large increases in serum macromolecules and may lead to a falsely low ESR in the higher ranges.

The micro-ESR only requires a few drops of capillary blood, which is useful in neonates. It correlates to the Westergren method for ESR values $0-15 \mathrm{~mm} / \mathrm{h}$, but for greater values a micro-ESR does not correlate directly. It increases with age: normal is $1-8 \mathrm{~mm} / \mathrm{h}$, with reports of up to 17 in the first 14 days of life. The micro-ESR is not influenced by gestational age, birth weight or sex in children. ${ }^{11}$

\section{Specimen handling}

The ESR is sensitive to a number of technical characteristics. It can be falsely decreased if blood is cooled rather than maintained at room temperature, is run more than $2 \mathrm{~h}$ after collection and/or clots (hence consumes fibrinogen) prior to placement in the tube. Equipment and environmental factors can also impact the ESR value. Orientation of the tube, rack and table is important because a tilted tube will increase the ESR. ${ }^{12}$ Conversely, vibration of the test apparatus ${ }^{8}$ or a short tube can lower it.

\section{INDICATIONS AND LIMITATIONS}

\section{In a child who is refusing to bear weight on his leg, does} a normal ESR rule out septic arthritis?

In situations in which a joint effusion is visualised, a normal ESR (between 0 and $20 \mathrm{~mm} / \mathrm{h}$ ) may support the diagnosis of reactive arthritis, a benign entity that requires little intervention, over that of septic arthritis and serve to limit further procedures. ${ }^{9}$ A minimally elevated ESR is of little help in distinguishing the two, but a significantly elevated ESR $(>40 \mathrm{~mm} / \mathrm{h})$ can be helpful. In a study of paediatric patients being evaluated for septic hip arthritis, ESR was $<40 \mathrm{~mm} / \mathrm{h}$ in only $44 \%$ of patients with septic arthritis compared with $86 \%$ of those with transient synovitis $(p<0.01) .^{9}$ Numerous attempts have been made to model the role of clinical parameters and laboratory values in predicting the likelihood of septic arthritis. In one study, when using a single laboratory test, both CRP and ESR were statistically significantly associated with having septic arthritis in children. ${ }^{9}$ Yet a model including a combination of variables (fever, refusal to bear weight, CRP $>2.0 \mathrm{mg} / \mathrm{dL}$, white blood cell
$>12000 \mathrm{~m}^{3}$ and ESR $>40 \mathrm{~mm} / \mathrm{h}$ ) best predicted septic arthritis (predicted probability 98\%).

In the absence of infection, does an elevated ESR necessarily mean a patient has a rheumatological disease? No. In addition to infection and rheumatological processes, the ESR can be elevated in numerous disease states (table 3 ). Significantly elevated ESRs ( $\geq 40$ or $\geq 100$, depending on the study) can occur in malignancy (lymphoma, acute childhood leukaemia and metastatic tumours ${ }^{2}$ ), post-transplantation states, interstitial lung disease, inflammatory bowel disease (IBD), ${ }^{5}$ renal failure, ischaemic tissue injury, trauma and pregnancy (and intrauterine fetal death). In one study of children (average age $\leq 10$ years) with ESR $\geq 100 \mathrm{~mm} / \mathrm{h}$, underlying diagnoses broke down as follows: $49.5 \%$ infection, $26.3 \%$ connective tissue disease, $12.1 \%$ malignancy and $8.1 \%$ renal disease. ${ }^{15}$ In the absence of obvious infection or other aetiology, an elevated ESR with abdominal symptoms and/or joint pain should raise suspicion for IBD and Coeliac disease. $^{5}$

Once a rheumatological disease has been diagnosed, is it helpful to order subsequent ESRs?

For those diseases in which it is elevated at diagnosis, the ESR can be helpful in assessing for disease

Table 3 Conditions in which erythrocyte sedimentation rate (ESR) is elevated ${ }^{2} 5712 \mathrm{w} 2$

$\begin{array}{ll}\text { Infectious } & \\ \text { Oncological } & \\ \text { Renal } & \text { Glomerulonephritis } \\ & \text { Renal failure } \\ \text { Rheumatological } & \text { Acute rheumatic fever } \\ & \text { Chronic recurrent non-infectious multifocal } \\ & \text { osteomyelitis* } \\ & \text { Juvenile dermatomyositis } \\ & \text { Juvenile idiopathic arthritis (polyarticular, systemic) } \\ & \text { Periodic fever syndromes } \\ & \text { Sarcoid disease } \\ & \text { Some vasculitides: } \\ & \text { Small vessel vasculitis: } \\ & - \text { Granulomatosis with polyangitis } \\ & - \text { Isolated childhood primary angiography-negative } \\ & \text { central nervous system vasculitist } \\ & - \text { Henoch-Schönlein purpura } \\ & \text { Medium vessel vasculitis: } \\ & - \text { Polyarteritis nodosa } \\ & - \text { Kawasaki disease } \\ & \text { Other vasculitides: } \\ & - \text { Behçet disease } \\ & \text { Systemic lupus erythematosus } \\ & \text { Congestive heart failure } \\ \text { Inflammatory bowel disease/Coeliac disease } & \text { Interstitial lung disease } \\ \text { Obesity } \\ \text { Post-transplantation } \\ \text { Pregnancy }\end{array}$


improvement because it tends to normalise with remission and rise with flares. It may be used to monitor disease activity in many diseases (table 3). ESR levels are particularly valuable in the course of systemic lupus erythematosus where they closely parallel disease activity. ${ }^{5}$ Elevated ESR is one of the minor diagnostic criteria for acute rheumatic fever (ARF) (Jones criteria). ${ }^{5}$ In ARF, the decision to taper non-steroidal anti-inflammatory drug therapy is based upon improvement in the ESR. As in many vasculitides, the ESR is elevated in active Kawasaki disease (KD) and decreases with KD resolution. Importantly, in patients with KD treated with IVIg, levels will no longer correlate with disease activity as IVIg itself increases the ESR. ${ }^{4}$ While systemic inflammation does not occur in all types of juvenile idiopathic arthritis (JIA), the ESR is elevated in active polyarticular and systemic JIA. As a matter of fact, the Wallace criteria for JIA remission include a normal ESR as a necessary feature to reach inactivity. ${ }^{\text {w3 }}$

\section{In patients who are well appearing, can ESR be used to estimate the risk of worsening disease?}

An elevated ESR, along with other clinical signs and symptoms, may aid in diagnosing and monitoring disease activity as well as in prognosticating the risk of poor disease outcomes. Elevated ESR is associated with increased risk for the developing coronary artery disease. This may be due to atherogenesis driven by elevated fibrinogen levels. ${ }^{16}$ While this has been demonstrated in adult males, it may hold true in children as well. In adults with Hodgkin's lymphoma, an elevated ESR ( $>10 \mathrm{~mm} / \mathrm{h}$ in males and $>20 \mathrm{~mm} / \mathrm{h}$ in females) has been shown to be $87 \%$ sensitive and $79 \%$ specific for predicting relapse. ${ }^{17} \mathrm{~A}$ mildly elevated ESR $(\geq 28 \mathrm{~mm} / \mathrm{h})$ was associated with poor prognosis in patients who had a stroke. In adults with renal carcinoma, ESR of $\geq 54 \mathrm{~mm} / \mathrm{h}$ is an independent factor for poor prognosis. ${ }^{7}$ Plasma viscosity is another indirect acute-phase/delayed acute-phase reactant. It can parallel ESR and is also affected by plasma proteins and fibrinogen, but without the variations related to age, sex, haemoglobin and timing of the test itself. ${ }^{\mathrm{w} 1}$ It is affected by plasma proteins, such as fibrinogen, and also physical activity. Unlike the ESR, it is affected by physical activity and more by chronic inflammatory conditions and less responsive to acute changes in inflammation. Technically it is more difficult to perform than ESR and it has fallen out of favour. There are few studies regarding the comparison of plasma viscosity and ESR in paediatrics. ${ }^{18}$

\section{In a child presenting with features concerning for} rheumatic disease, does a normal ESR rule it out?

A normal ESR never rules out inflammatory disease, but it may decrease the suspicion. Positive and negative predictive values are not available regarding specific thresholds of ESR values. Even when active, some
Box 3 Rheumatological disease not typically associated with an elevated ESR $^{5}$

Juvenile idiopathic arthritis*

- Angiography-positive cPACNS (moderate-large vessel vasculitis)

- Linear morphea

- Macrophage activation syndrome

Takayasu arteritis (large vessel vasculitis) $\dagger$

Uveitis

*Except systemic and polyarticular subtypes of juvenile idiopathic arthritis.

tMay be elevated in active disease, but not always.

rheumatological conditions are not necessarily associated with an elevated ESR (box 3), in particular the oligoarticular, psoriatic and enthesitis-related forms of JIA. While the ESR may be elevated in the large vessel vasculitis, Takayasu arteritis (TA), it may, counterintuitively, be normal in active TA. Other rheumatic conditions are associated with only mildly increased ESR (eg, chronic recurrent multifocal noninfectious osteomyelitis). Notably, a normal ESR is far from reassuring in macrophage activation syndrome. In this severe condition with widespread inflammation, the ESR drops precipitously with increased disease activity because of worsening hypofibrinogenaemia.

\section{In a well-appearing child, does an elevated ESR always} signify an active source of disease?

An elevated ESR is not necessarily an indicator of infection or inflammation, as a variety of factors might be at play in a patient with an elevated ESR.

- The ESR may be elevated by obesity alone. The mean ESR was both clinically $(13 \mathrm{~mm} / \mathrm{h})$ and statistically $(\mathrm{p}=0.008)$ higher in otherwise healthy obese adults relative to their non-obese peers (33 vs $20 \mathrm{~mm} / \mathrm{h}$ ). ${ }^{\mathrm{w} 4}$ Ciccone examined this relationship in obese and overweight children and found a statistically significant correlation between body mass index (BMI) and ESR $(\mathrm{p}<0.001)$, wherein ESR rises linearly with BMI (see table 3). ${ }^{19}$ Importantly, while such patients may not have another underlying inflammatory disease, their obesity itself may result in an inflammatory state. ${ }^{\mathrm{w} 5}$

- Race also affects the ESR. Even with correction for haemoglobin concentration, other chronic diseases and age, the baseline ESR is $2-13 \mathrm{~mm} / \mathrm{h}$ higher in individuals of African descent than in individuals of other races. ${ }^{16}$

Other factors may affect the baseline ESR but would not necessarily explain elevations above the normal range:

- ESR values are higher in females than in males over a certain age (see table 4). Studies demonstrate that castration results in elevation of the ESR, suggesting that androgens may suppress it. ${ }^{8}$ In pregnancy, the ESR 
Table 4 Erythrocyte sedimentation rate (ESR) values by age ${ }^{4} 12$

\begin{tabular}{ll}
\hline Age & ESR (mean, $\mathrm{mm} / \mathrm{h})$ \\
\hline $0-1$ month & $0-2$ \\
1 month-12 years & $\leq 10-20$ \\
$>12$ years & \\
Male & $\leq 15$ \\
Female & $\leq 20$ \\
\hline
\end{tabular}

steadily rises and then falls 3-4 weeks postpartum, presumably due to the influence of oestrogen. ${ }^{11}$ Conversely, there seems to be an inverse relationship with oestrogen levels and ESR in older women. Bottiger and Svedberg demonstrated that while ESR rises in both sexes with age, the rate of rise is greater in women following menopause. $^{10}$

- One should also consider the medications the patient is taking, as they can falsely decrease or increase the ESR relative to its value in the individual or underlying disease state (see table 5).

- Age affects the ESR value. Most laboratories consider an ESR between 0 and $20 \mathrm{~mm} / \mathrm{h}$ to be in the normal range after the neonatal period ${ }^{20}$ (see table 4$) .{ }^{4}$ Despite the general acceptance of these values, there are no publications in which normal ESR values by age in paediatrics have been evaluated. Although it had been commonly accepted that the mean ESR rose with age, in 1951, Wilhelm convincingly demonstrated this finding. He showed that the mean ESR in people in their third decade of life was $8.3 \mathrm{~mm} / \mathrm{h}$ (95\% CI 6.5 to 10.1$)$ while that of those in their eighth decade was $18.3 \mathrm{~mm} / \mathrm{h}$ (95\% CI 17.3 to 22.4). His results, and others like his, suggest that age should be considered when interpreting an ESR value. ${ }^{\text {w6 }}$

The degree of ESR elevation is important in determining potential aetiologies. If it is moderately elevated $\left(20-30 \mathrm{~mm} / \mathrm{h}^{4}\right)$, one should consider the aforementioned factors. If it is extremely elevated $\left(>100 \mathrm{~mm} / \mathrm{h}^{2}{ }^{15}\right)$, one should have a higher suspicion for underlying infection or inflammation.

Table 5 Drugs affecting erythrocyte sedimentation rate (ESR) level $\left.\right|^{2}$

\begin{tabular}{ll}
\hline Decreased ESR & Elevated ESR \\
\hline Asparaginase & Dextran \\
Cortisone & Heparin \\
High-dose salicylates & IVIG \\
Low molecular weight dextran & Oral contraceptives \\
Non-steroidal anti-inflammatory drugs & \\
Valproic acid & \\
\hline
\end{tabular}

\section{TOPICS FOR FURTHER RESEARCH}

The ESR is a commonly used, low-technology blood test that can provide valuable clinical information. In conjunction with physical findings and other laboratory values, the ESR value can be used to screen for disease or disease complications; aid in disease diagnosis; monitor disease activity and response to therapy; and aid in prognosis of disease. In the future, it would be important to evaluate how ESR values vary by age to establish normal paediatric ranges. Furthermore, it would be interesting to determine the pathogenesis of elevated ESR in obese individuals.

\section{Clinical bottom line}

Erythrocyte sedimentation rate (ESR) is an important tool to assess for inflammation.

- Normal ESR should not be used alone to rule out disease, but can reassure against active disease when clinical suspicion for infection, inflammation or malignancy is low.

- ESR may be superior to other acute-phase reactants for monitoring inflammation in chronic conditions because it rises and falls slowly.

\section{Test your knowledge}

1. True or false: erythrocyte sedimentation rate (ESR) is a direct measurement of inflammation

2. ESR is elevated in which of the following:
A. Hypofibrinogenaemia
B. Sickle cell disease
C. Osteomyelitis

3. True or false: ESR can be used alone to rule out neoplasm or infection

4. Which of the following increases the ESR?
A. Anaemia
B. Polycythaemia
C. Haemolytic anaemia

5. ESR is elevated in which conditions? (May choose more than one answer)
A. Advanced age
B. Males
C. Periodic fever syndromes

Answers are at the end of the references

Supplementary appendices A-B are available on the journal website.

Contributors The Archives of Disease in Childhood had the idea for this article. ESR performed the literature search. ESR and MAL wrote the article. MAL is the guarantor for the article.

Competing interests None. 
Provenance and peer review Commissioned; externally peer reviewed.

\section{REFERENCES}

1 Breda L, Nozzi M, De Sanctis S, et al. Laboratory tests in the diagnosis and follow-up of pediatric rheumatic diseases: an update. Semin Arthritis Rheum 2010;40:53-72.

2 Sox HC, Liang MH. The erythrocyte sedimentation rate. Guidelines for rational use. Ann Intern Med 1986;104:515-23.

3 Osei-Bimpong A, Meek JH, Lewis SM. ESR or CRP? A comparison of their clinical utility. Hematology 2007;12:353-7.

4 Long SS, Pickering LK, Prober CG. Long: principles and practice of pediatric infectious diseases. Elsevier Saunders, 2012:1400-2.

5 Cassidy JT, Petty RE, Lindsley CB, et al. Textbook of pediatric rheumatology. Philadelphia, PA: Elsevier, 2011:244, 280, 340, 398, 461, 514, 606, 646, 678, 693.

6 Murray RDB, Botham KM, Kennelly PJ, et al. Harpers illustrated biochemistry. New York, NY: McGraw-Hill Medical, 2012:583-4.

7 Brigden M. The erythrocyte sedimentation rate. Still a helpful test when used judiciously. Postgrad Med 1998;103:257-62, 72-4.

8 Bedell SE, Bush BT. Erythrocyte sedimentation rate. From folklore to facts. Am J Med 1985;78:1001-9.

9 Caird MS, Flynn JM, Leung YL, et al. Factors distinguishing septic arthritis from transient synovitis of the hip in children. A prospective study. J Bone Joint Surg Am 2006;88:1251-7.

10 Bottiger LE, Svedberg CA. Normal erythrocyte sedimentation rate and age. Br Med J 1967;2:85-7.

11 McPherson RA. Mcpherson: Henry's clinical diagnosis and management by laboratory methods. New York, NY: Elsevier, 2011:519-21.
12 Lascari AD. The erythrocyte sedimentation rate. Pediatr Clin North Am 1972;19:1113-21.

13 Reinhart WH, Nagy C. Albumin affects erythrocyte aggregation and sedimentation. Eur J Clin Invest 1995;25:523-8.

14 Jou JM, Lewis SM, Briggs C, et al. ICSH review of the measurement of the erythocyte sedimentation rate. Int J Lab Hematol 2011;33:125-32.

15 Abbag FI, Al Qahtani JM. Extreme elevation of the erythrocyte sedimentation rate in children. Ann Saudi Med 2007;27:175-8.

16 Saadeh C. The erythrocyte sedimentation rate: old and new clinical applications. South Med J 1998;91:220-5.

17 Ray GR, Wolf PH, Kaplan HS. Value of laboratory indicators in Hodgkin's disease: preliminary results. Natl Cancer Inst Monogr 1973;36:315-23.

18 Kesmarky G, Kenyeres P, Rabai M, et al. Plasma viscosity: a forgotten variable. Clin Hemorheol Microcirc 2008;39:243-6.

19 Ciccone MM, Miniello V, Marchioli R, et al. Morphological and functional vascular changes induced by childhood obesity. Eur J Cardiovasc Prev Rehabil 2011;18:831-5.

20 Dawson JB. The E.S.R. in a new dress. Br Med J 1960;1:1697-704.

\section{Answers to the quiz}

1. False

2. C

3. False

4. A

5. A and C 